\title{
Immunological aspects of REIC/Dkk-3 in monocyte differentiation and tumor regression
}

\author{
MASAMI WATANABE ${ }^{1,2}$, YUJI KASHIWAKURA ${ }^{1}$, PENG HUANG $^{2}$, KAZUHIKO OCHIAI $^{1}$, \\ JUNICHIRO FUTAMI ${ }^{1}$, SHUN-AI LI ${ }^{1}$, MUNENORI TAKAOKA ${ }^{1}$, YASUTOMO NASU ${ }^{2}$, \\ MASAKIYO SAKAGUCHI ${ }^{3}$, NAM-HO HUH ${ }^{3}$ and HIROMI KUMON ${ }^{1,2}$ \\ ${ }^{1}$ Innovation Center Okayama for Nanobio-Targeted Therapy, ${ }^{2}$ Urology, ${ }^{3}$ Cell Biology, Graduate School of Medicine, \\ Dentistry and Pharmaceutical Sciences, Okayama University, Okayama 700-8558, Japan
}

Received August 29, 2008; Accepted November 3, 2008

DOI: 10.3892/ijo_00000191

\begin{abstract}
The REIC/Dkk-3 gene has been reported to be a tumor suppressor and the expression is significantly downregulated in a broad range of cancer cell types. The protein is secretory, but the physiological function remains unclear. This study demonstrated that recombinant REIC/Dkk-3 protein induced the differentiation of human $\mathrm{CD} 14^{+}$monocytes into a novel cell type $\left({ }^{\mathrm{REIC} / \mathrm{Dkk}-3} \mathrm{Mo}\right) .{ }^{\mathrm{REIC} / \mathrm{Dkk}-3} \mathrm{Mo}$ resembles immature dendritic cells generated with IL-4 and GM-CSF. Both these cell populations exhibit similar proportions of $\mathrm{CD} 11 \mathrm{c}^{+}, \mathrm{CD} 40^{+}$, $\mathrm{CD}^{+} 6^{+}$and HLA-DR ${ }^{+}$cells and endocytic capacity, but REIC/Dkk-3 Mo is negative for CD1a antigen. An analysis of the signal transducers and activators of transcription (STAT) pathways revealed that REIC/Dkk-3 induces phosphorylation of STAT 1 and STAT 3. Furthermore, intratumoral administration of REIC/Dkk-3 protein significantly suppressed tumor growth with $\mathrm{CD} 11 \mathrm{c}^{+}$and $\mathrm{CD} 8^{+}$(dendritic and killer $\mathrm{T}$ cell marker, respectively) cell accumulation and enhanced anticancer cytolytic activity of splenocytes. These data indicated a cytokine-like role of REIC/Dkk-3 protein in monocyte differentiation that might be exploited therapeutically.
\end{abstract}

\section{Introduction}

The REIC (reduced expression in immortalized cells) gene was previously cloned and it is identical to the Dickkopf-3 (Dkk-3) gene, which is a member of the Dickkopf gene family (1). The expression of REIC/Dkk-3 is significantly down-regulated in a broad range of cancer cell lines and clinical tumors (1-6). The forced expression of REIC/Dkk-3, using a

Correspondence to: Dr Masami Watanabe, Innovation Center Okayama for Nanobio-Targeted Therapy, Okayama University, 2-5-1 Shikata-cho, Okayama 700-8558, Japan

E-mail: mwcorrespondence@gmail.com

Key words: REIC, Dkk-3, monocyte, dendritic cell, anti-cancer immune plasmid vector, inhibits cell growth in HeLa and liver cancer cell lines (3). An adenovirus vector carrying REIC/Dkk-3 selectively induces apoptosis in prostatic and testicular cancer cells but not in non-cancer cells, through the activation of c-Jun-NH2-kinase (JNK) and c-Jun $(4,5)$. A recent study using malignant mesothelioma cells indicated that adenovirus mediated REIC/Dkk-3 overexpression triggers significant induction of apoptosis via endoplasmic reticulum (ER) stress (6). Ad-REIC treatment also inhibits the expression of Id-1, which works in cell cycle progression and anti-apoptosis (7). Therefore, accumulating evidence suggests that intracellular overexpression of REIC/Dkk-3 plays a distinct role in apoptotic induction and anti-oncogenesis. However, there have been few reports investigating the physiological functions of secretory or exogenous REIC/Dkk-3 protein. Although REIC/Dkk-3 is a member of the Dickkopf protein family known to interfere with $\mathrm{Wnt} / \mathrm{B}$-catenin signaling via Wnt receptors $(1,2,8)$, some studies have demonstrated that REIC/Dkk-3 does not affect the Wnt/ß-catenin signaling in these conditions $(2,9)$. Until now, the molecular function and mechanism by which REIC/Dkk-3 protein exogenously acts on specific cell types remained unknown. This study presents novel evidence showing the immunological role of REIC/Dkk-3 protein in monocyte differentiation and in vivo tumor regression.

\section{Materials and methods}

Preparation of recombinant human REIC/Dkk-3 protein. Human REIC/Dkk-3 stable expression clones in CHO cells were established by electroporated transfection of the plasmids encoding the full length human REIC/Dkk-3 gene with a histidine tag and a puromycin-resistant gene. A total of $10^{7}$ cells were cultured in 11 of $\mathrm{CHO}$ protein-free medium (C5467, Sigma, St. Louis, MO) with $2 \mathrm{mM}$ L-glutamine and $8 \mu \mathrm{M}$ puromycin at $37^{\circ} \mathrm{C}$ in $5 \% \mathrm{CO}_{2}$ atmosphere for 1 week with gentle shaking. Using the TALON Resin (no. 635501, Clontech Laboratories, Mountain View, CA) in the culture supernatant, the histidine-tagged human REIC protein was collected and then subjected to Fast Protein Liquid Chromatography (MonoQ5/50GL column, GE Healthcare, Buckinghamshire, UK). The purified human REIC protein was dialyzed into 
phosphate-buffered saline (PBS) for stock and use and the stock solutions of the protein were maintained at $-80^{\circ} \mathrm{C}$ until use.

Preparation of human monocytes. Human PBMC (peripheral blood mononuclear cell) were prepared from the blood of healthy donors by the standard procedure of Ficoll-Paque centrifugation. To purify the monocytes, the PBMC were resuspended in LGM-3 medium (Lonza, Walkersville, MD) and incubated in $10 \mathrm{~cm}$ plastic dish at $37^{\circ} \mathrm{C}$ for $2 \mathrm{~h}$. After the incubation, the adherent cells were used for the monocytes. In some experiments, the CD14 positive monocytes were isolated from the PBMC using $\mathrm{CD}_{14}{ }^{+}$magnetic activated cell sorting microbeads (Miltenyi Biotec, Bergisch Gladbach, Germany), according to the manufacturer's instructions.

Culture conditions. Purified monocytes were cultured either in LGM-3 medium alone or in the presence of recombinant human REIC/Dkk-3 protein ( $10 \mu \mathrm{g} / \mathrm{ml}$, if not indicated). As a positive control for the differentiation of dendritic cells, the monocytes were cultured in LGM-3 medium supplemented with interleukin-4 (IL-4, 2 ng/ml, R\&D Systems, Minneapolis, $\mathrm{MN})$ and granulocyte-macrophage colony-stimulating factor (GM-CSF, 2 ng/ml, R\&D Systems) $(10,11)$. The cells were cultured in a final volume of $10-15 \mathrm{ml}$ at $37^{\circ} \mathrm{C}$ in humidified incubators containing air with $5 \% \mathrm{CO}_{2}$.

Western blot analysis. The total protein from treated cells or tumor tissue was extracted and Western blotting was done as described previously $(4,12)$. Proteins were identified with the use of the following primary antibody at 1000x dilution; rabbit polyclonal anti-human REIC/Dkk-3 antibody raised in this laboratory (4), anti-phospho-STAT 1 (Try701) (no. 9171, Cell Signaling Technology, Inc. Danvers, MA), antiphospho-STAT 3 (Try705) (no. 9131), anti-phospho-STAT 6 (Try641) (no. 9361).

Flow cytometric analysis. The floating and adherent cells were combined and approximately $5 \times 10^{5}$ cells were incubated with the following PE-conjugated antibodies $(5 \mathrm{x}$ diluted in $100 \mu \mathrm{l}$ PBS) for $60 \mathrm{~min}$ on ice; CD11c (12-0116, eBioscience, San Diego, CA), CD14 (12-0149), CD1a (12-0019), CD40 (12-0409), CD80 (12-0809), CD83 (12-0839), CD86 (12-0869), HLA-DR (12-9956). Incubation with PE-conjugated immunoglobulin $\mathrm{G}$ ( $\mathrm{IgG}$ ) isotype-matched irrelevant antibodies (12-4714, 12-4732, eBioscience) were used as negative controls. After staining, $10^{4}$ cells were acquired on a FACSCalibur flow cytometer (Becton-Dickinson) and analyzed using the CellQuest software program (BectonDickinson). To examine the endocytic capacity of the cells, $50 \mu \mathrm{g}$ of FITC-conjugated dextran (FITC-DX; FD40, Sigma, St. Louis, MO) was added to $0.5 \mathrm{ml}$ of cell suspension $\left(5 \times 10^{6}\right.$ cells $\left./ \mathrm{ml}\right)$. The cells were then incubated at $4^{\circ} \mathrm{C}$ or $37^{\circ} \mathrm{C}$ for $1 \mathrm{~h}$ and analyzed by a flow cytometer.

Animal experiments. The RM9 mouse prostate cancer cell line was kindly provided by Dr T.C. Thompson (The University of Texas, Houston, TX) (13) and used in the animal experiments. To investigate the anti-tumor effects of REIC/Dkk-3 protein, $1 \times 10^{6}$ RM9 cells were subcutaneously injected into the left thigh of C57BL/6 adult male mice with $0.1 \mathrm{ml}$ PBS. On day 7 , when the tumor volume reached $\sim 300 \mathrm{~mm}^{3}$, each tumor was treated intratumorally with REIC protein or PBS alone as shown in Fig. 3A. In the other experiments shown in Fig. 4A, the orthotopic prostate cancer model with pre-established lung metastases was used $(14,15)$ and the Ad-REIC or Ad-LacZ prepared as previously described (4) was injected into the orthotopic tumor on day 7. The injection of the agents was targeted to the center and periphery of each mass to deliver the agent diffusely and the tumor volume was determined as described previously (12).

In vitro cytolytic assays. To prepare the splenocytes, the spleen from each mouse was dissociated with glass slides and passed through a $70-\mu \mathrm{m}$ cell strainer to obtain the cellular fraction. After lysing the red blood cells with ACK lysing buffer (Cambrex Bioscience, Walkersville, MD), the splenocytes were resuspended in $2 \%$ fetal bovine serum supplemented DMEM (Dulbecco's modified Eagle's medium, Invitrogen, Carlsbad, CA) and used for the cytolytic assays as an effector. RM9 cells were used for the target and the splenocytes were cultured with RM9 cells preseeded in a 96-well round bottom plate in duplicate in a ratio of effector/target of 100:1, 50:1, $25: 1,12.5: 1$. A total of $5 \times 10^{3}$ target cells was applied per well. Supernatants were collected and lactate dehydrogenase released from lysed RM9 cells was measured using a nonradioactive cytotoxicity assay (CytoTox 96, Promega, Madison, WI). The percentage of lysed cells was calculated according to the following formula: (experimental release - effector spontaneous release - target spontaneous release)/(target maximal release - target spontaneous release) $\mathrm{x} 100 \%$.

Histological procedure. Immunohistochemical staining was performed as previously described (12), using anti-CD11c and CD8 antibody (no. 550375 and no. 550281, respectively, BD Pharmingen, San Diego, CA) for the first antibody. To detect apoptotic cells in the tumor tissue, terminal deoxynucleotidyl transferase-mediated UTP end-labeling (TUNEL) assay was done using an In situ Cell Death Detection Kit, Fluorescein (Roche, Penzberg, Germany).

Statistical analysis. The data are shown as the mean $\pm \mathrm{SE}$. Unpaired Student's t-test was performed for the statistical analysis between the two groups and the difference was considered significant at $\mathrm{p}<0.05$.

\section{Results}

REIC/Dkk-3 protein induces differentiation of monocytes into dendritic-cell like features. Full-length human REIC/Dkk-3 protein was produced in $\mathrm{CHO}$ cells and the purity was confirmed to be $>95 \%$ by gel electrophoresis (Fig. 1A). Highly purified monocytes from PBMC were cultured in the presence of recombinant REIC/Dkk-3 protein (subsequently designated ${ }^{\mathrm{REIC} / \mathrm{Dkk}-3} \mathrm{Mo}$ ) or with established protocols of dendritic cell differentiation by the addition of IL-4 and GM-CSF (subsequently designated IL-4+GM-CSFDC). During the first 2 days of incubation with REIC/Dkk-3 protein, approximately half of the ${ }^{\mathrm{REIC} / \mathrm{Dkk}-3} \mathrm{Mo}$ displayed cellular elongation (Fig. 1B). The change of cell morphology was 
A

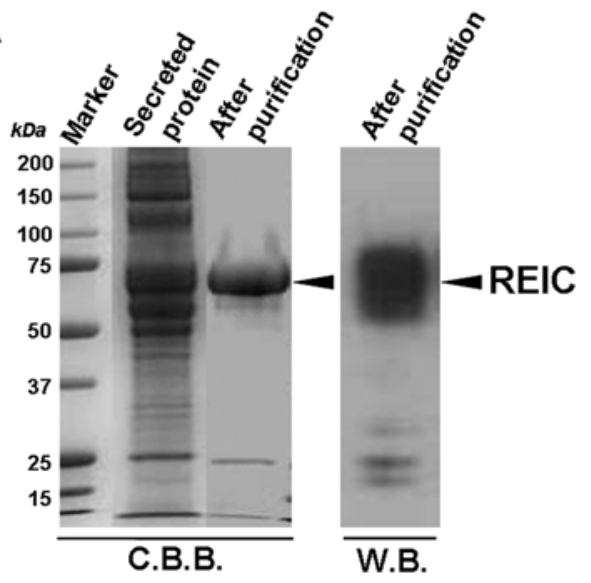

B

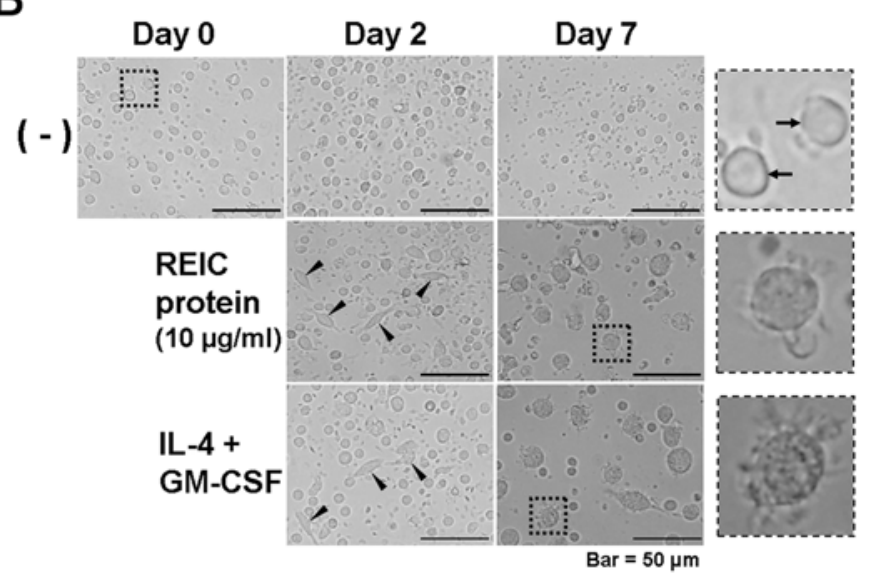

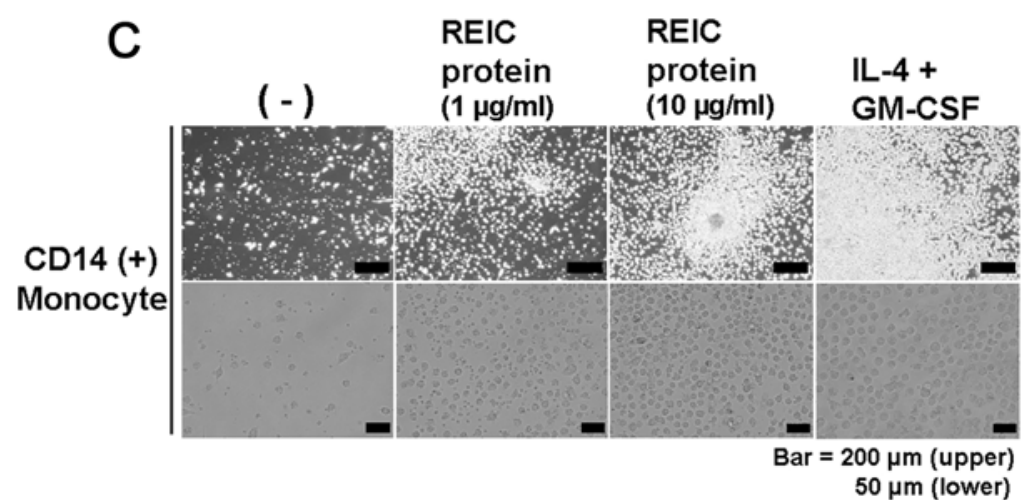

Figure 1. (A) The purity of recombinant human REIC/Dkk-3 protein was confirmed by SDS-PAGE with Coomassie brilliant blue (CBB) staining. The band was also verified to be REIC/Dkk-3 protein by Western blotting (WB). (B) Highly purified human monocytes were treated with medium alone or REIC/Dkk-3

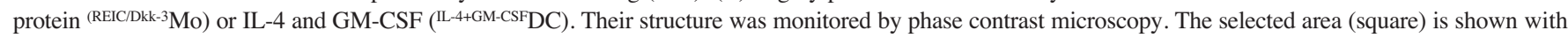
magnification. The arrowhead indicates the elongated monocyte on day 2 . The arrow indicates the monocyte without treatment. (C) The CD14 ${ }^{+}$monocytes were cultured with the indicated treatment and were observed on day 14.

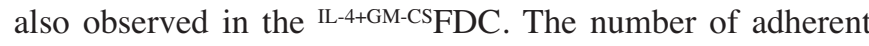
REIC/Dkk-3Mo was markedly reduced after 5 days in culture and by day 7 most of the cells were suspended in the medium (Fig. 1B). The population of ${ }^{\mathrm{REIC} / \mathrm{Dkk}-3}$ Mo indicates dendritic-cell

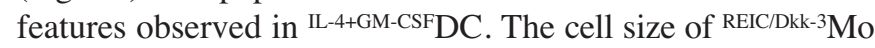
is larger in comparison to the untreated monocytes on day 0 . As for the monocytes treated in medium alone for 7 days, there were few cells with dendritic-cell like features and most of the monocytes appeared shrunken in comparison to the monocytes on day 0 . To further verify the effects of REIC/ Dkk-3 protein on the monocyte differentiation, human $\mathrm{CD} 14^{+}$monocytes were isolated from PBMC. In the CD14 ${ }^{+}$ monocytes, the differentiation into the dendritic phenotype occurred dose-dependently after incubation with REIC/Dkk-3 protein (Fig. 1C). In the CD14 negative monocytes, no dendritic-like cells were observed after REIC/Dkk-3 incubation (data not shown).

The activation of STAT signaling is responsible for the differentiation of a variety of blood cells $(16,17)$. Therefore, we analyzed whether REIC/Dkk-3 protein causes activation of STAT signaling by phosphorylation. The level of phosphorylation of STAT 1 and STAT 3 was significantly up-regulated in ${ }^{\mathrm{REIC} / \mathrm{Dkk}-3}$ Mo after 6-h incubation, in comparison to the monocytes cultured in medium alone (Fig. 2A). The phos- phorylation of STAT 3 was observed at $0.5 \mathrm{hr}$ after the exposure. STAT 6, which is a signal transducer of IL-4, was not phosphorylated by treatment with REIC/Dkk-3 protein during the incubation period.

Phenotypic characterization of the monocytes cultured with REIC/Dkk-3 protein. The influence of REIC/Dkk-3 protein on the differentiation of monocytes into the dendritic cell phenotype was then verified $(10,11)$. The ${ }^{\text {REIC/Dkk-3 Mo on }}$ day 7 was analyzed for specific surface markers by flow cytometry and ${ }^{\mathrm{L}-4+\mathrm{GM}-\mathrm{CSF} D C}$ was used as the positive control for immature dendritic cells (Fig. 2B). In the monocytes cultured with medium alone for 7 days, propidium iodide (PI) staining in flow cytometry showed $<5 \%$ of the cells were viable (data not shown). The proportion of CD11 $\mathrm{c}^{+}$was similar between the ${ }^{\mathrm{REIC} / \mathrm{Dkk}-3} \mathrm{Mo}$ and ${ }^{\mathrm{IL}-4+\mathrm{GM}-\mathrm{CSF}} \mathrm{DC}$ populations. A lower proportion of $\mathrm{CD} 40^{+}$and $\mathrm{CD} 80^{+}$cells were observed

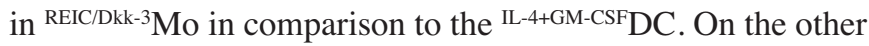
hand, the proportion of $\mathrm{CD}^{+} 6^{+}$, $\mathrm{HLA}-\mathrm{DR}{ }^{+}$and $\mathrm{CD} 14^{+}$cells in REIC/Dkk-3 Mo was significantly higher in comparison to the IL-4+GM-CSFDC. The CD1a surface antigen was positive in the

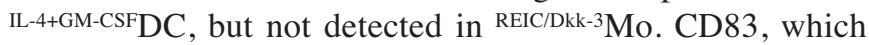
is a marker for mature dendritic cells, was negative in both cell populations. The endocytic capacity of these cell types 


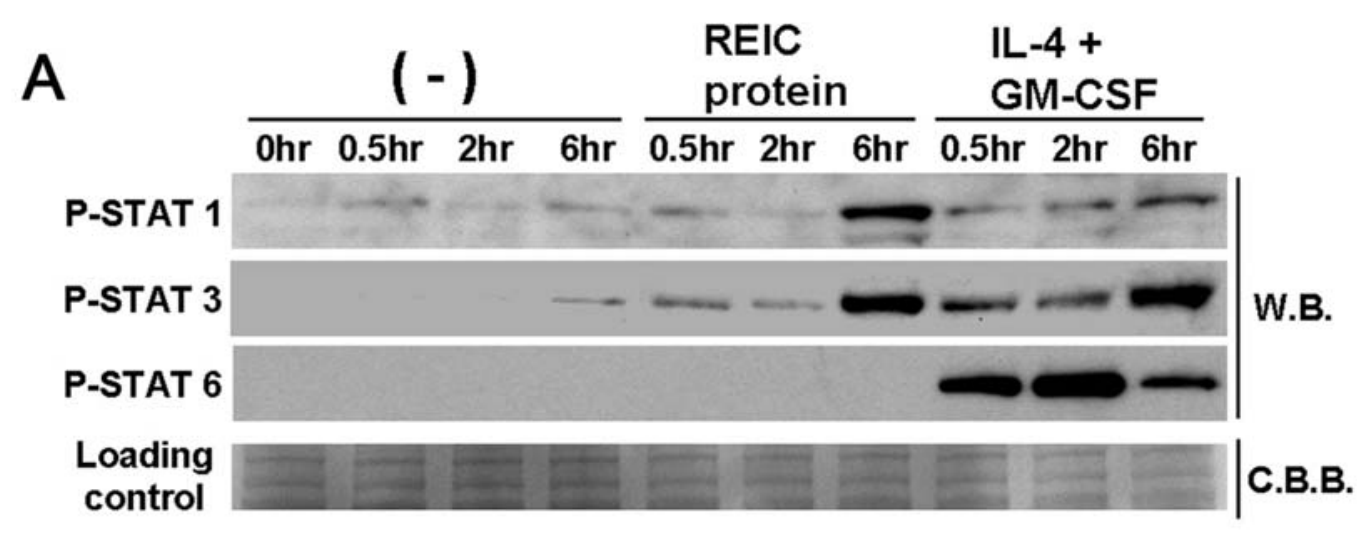

B
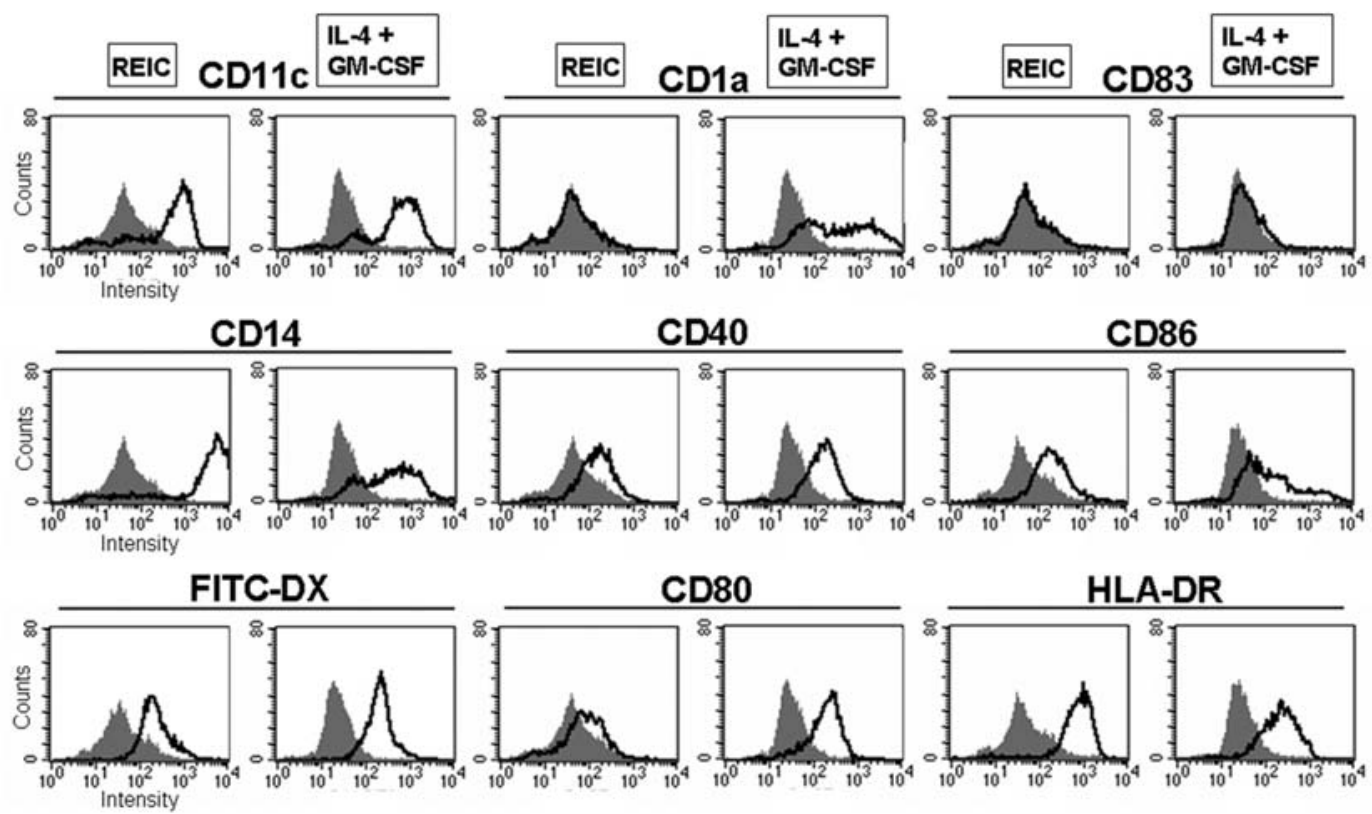

Figure 2. (A) REIC/Dkk-3 protein induces phosphorylation of STAT 1 and STAT 3 in REIC/Dkk-3Mo. The monocytes were incubated with REIC/Dkk-3 protein $(10 \mu \mathrm{g} / \mathrm{ml})$ or IL-4+GM-CSF for the time periods indicated. The levels of phosphorylation were determined by Western blotting using antibodies specific for the phosphorylated, activated forms. A Coomassie brilliant blue stained gel is shown in the bottom panel as a loading control. (B) Phenotypic characterization of REIC/Dkk-3 Mo. Purified monocytes were incubated with REIC/Dkk-3 protein $(10 \mu \mathrm{g} / \mathrm{ml})$ or IL-4+GM-CSF for 7 days. The cells were analyzed by flow cytometry and the proportion of positive cells is indicated for each surface antigen. The endocytosis of FITC-labeled dextran particles was also analyzed and the result is indicated in the FITC-DX panel.

was further evaluated by measuring the uptake of FITClabeled dextran particles. After 7 days of culture with REIC/Dkk-3 protein or IL-4+GM-CSF, the cells were exposed to FITC-DX for $1 \mathrm{~h}$ at either $4^{\circ} \mathrm{C}$ (a background control for non-specific binding) or $37^{\circ} \mathrm{C}$ (active uptake). The internalization of FITC-DX by ${ }^{\text {REIC/Dkk-3 Mo was equivalent }}$ to that of ${ }^{\mathrm{IL}-4+\mathrm{GM}-\mathrm{CSF} D C \text {. }}$

Intratumoral administration of REIC/Dkk-3 protein suppressed tumor growth in RM9 subcutaneous tumor model. The elevated expression of dendritic-cell markers with dendritic cell-like features indicated that the population of ${ }^{\mathrm{REIC} / \mathrm{Dkk}-3} \mathrm{Mo}$ might possess immunological capacities such as antigen presentation $(10,11)$. Since REIC/Dkk-3 is a tumor suppressor gene and significantly down-regulated in a broad range of cancer cells (1-6), the gene product should also have anticancer functions. In order to investigate the anti-cancer effects of REIC/Dkk-3 protein, the in vivo experiment shown in Fig. 3A was performed. A significant suppression of tumor growth was observed on day 12 and 14 in the REIC/Dkk-3 treated group (Fig. 3B). A histopathological analysis with hematoxylin and eosin staining revealed massive degeneration of the tissues in the REIC/Dkk-3 injected tumors, but not in those treated with PBS (Fig. 3C). These findings were consistent with the tumor growth inhibition observed after the REIC/Dkk-3 treatment. Higher magnification in the injected sites revealed that the infiltration of many lymphocytes was observed within the area of the necrotic cancer cells with less hematoxylin-stained nuclei. Since the in vivo immunological activity of REIC/Dkk-3 protein was conceivable in terms of the differentiation of the dendritic cell-like phenotype, an immunohistochemical analysis of CD11 $\mathrm{c}^{+}$was performed in the tumor tissue specimens. A highly significant accumulation of CD11c positive cells was observed on day 21 in REIC/ 
B

A

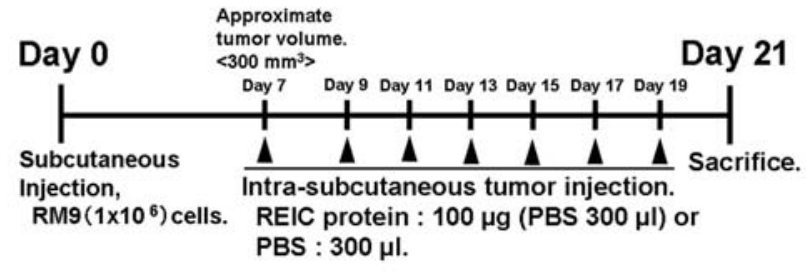

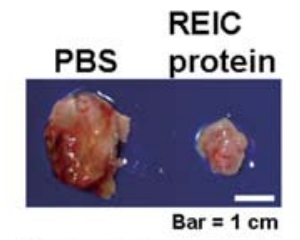

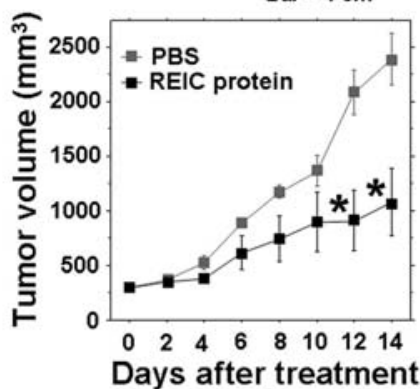

C

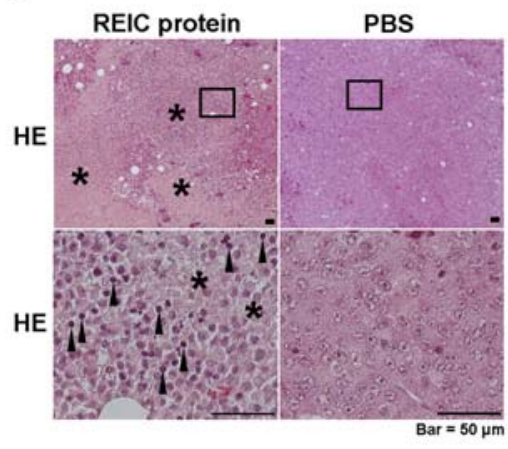

D

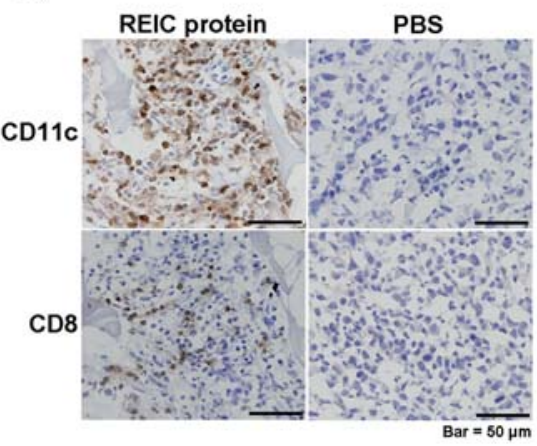

$\mathrm{E}$

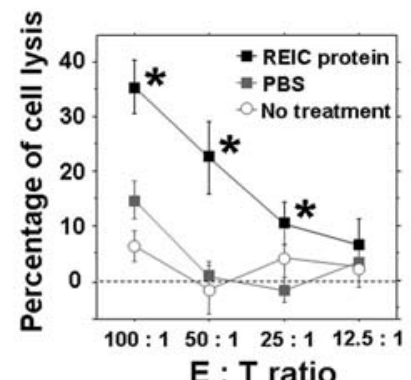

Figure 3. (A) Experimental schedule of treatment with REIC/Dkk-3 protein is shown. All mice were sacrificed on day 21. (B) Macroscopic view of excised RM9 tumors after the treatment is shown in the upper panel. The growth curve of the subcutaneous RM9 tumors is shown in the lower panel. Four mice were analyzed for each group. *A significant difference between REIC protein and PBS treatment. (C) Hematoxylin and eosin (HE) staining is shown in the indicated tumors. The selected area (square) in the upper panel is magnified in the lower panel. Arrowhead indicates a mononuclear cell typical of lymphocytes. Asterisk shows the necrotic area which is less stained with hematoxylin. (D) The infiltration of CD11 $\mathrm{c}^{+}$cells and CD8 $8^{+}$cells within the treated tumors was examined by immunohistochemistry. The CD11c and CD8 surface antigen was used for the marker of dendritic cell and killer T lymphocyte, respectively. (E) An in vitro RM9-cytolytic assay was performed using the splenocytes from each treated mouse. In the indicated effector/target (E:T) ratio, the percentage of the lysed cells was calculated. *A significant difference between REIC protein and PBS treatment. Four mice were analyzed for each group.

Dkk-3 treated subcutaneous tumors but not in the controls (Fig. 3D). In addition, the infiltration of CD8 positive killer $\mathrm{T}$ cells was also detected in the serial sections. In vitro cytolytic assays revealed the enhanced cytotoxic activity of splenocytes from REIC/Dkk-3 protein treated mice against the RM9 prostate cancer cells (Fig. 3E).

Overexpression of REIC/Dkk-3 protein in RM9 orthotopic prostate tumor suppressed the lung tumor formation in preestablished metastasis model. Adenovirus mediated REIC/ Dkk-3 overexpression was previously demonstrated to induce significant apoptosis in injected tumor sites and robust antitumor effects $(4-6,15)$. In vitro $\mathrm{REIC/Dkk-3}$ overexpression by Ad-REIC in cancer cells resulted in plenty of REIC/Dkk-3 protein secretion into the culture media (data not shown). Taken together, these findings lead to the hypothesis that the anti-cancer immune up-regulation by Ad-REIC treatment in primary tumor sites could trigger anti-tumor effects even at the distant tumor site. To test this hypothesis, an orthotopic RM9 prostate tumor model with pre-established lung metastasis was used (14) for the experiments as shown in Fig. 4A. The elevated expression of intratumoral REIC/Dkk-3 protein was first verified by Western blotting (Fig. 4B). The apoptotic induction by intratumoral Ad-REIC treatment was also confirmed by TUNEL staining (Fig. 4C). Consistently, the growth of orthotopic prostate tumors was strikingly inhibited 14 days after Ad-REIC injection in comparison to the controls (Fig. 4D). Moreover, the number of pre-established lung tumors significantly decreased in the Ad-REIC treated group (Fig. 4E). In vitro cytolytic assays revealed the enhanced anti-cancer immune activity of splenocytes from Ad-REIC treated mice against the RM9 prostate cancer cells (Fig. 4F).

\section{Discussion}

We currently elucidated the specific function of REIC/Dkk-3 protein in monocyte differentiation to a dendritic-cell phenotype. This is the first study to clearly demonstrate the exogenous action of REIC/Dkk-3 protein. The morphological features of ${ }^{\mathrm{REIC} / \mathrm{Dkk}-3} \mathrm{Mo}$ and the expression pattern of dendritic-cell markers on the surface are similar to those of IL-4+GM-CSFDC. These cell types also share the capacity of endocytosis, demonstrated by their FITC-DX up-take. How-

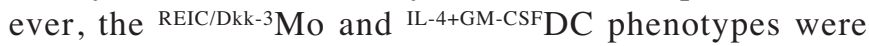
distinctly different in terms of the expression of CD1a and CD14 surface markers. In addition, the expression pattern of 
A
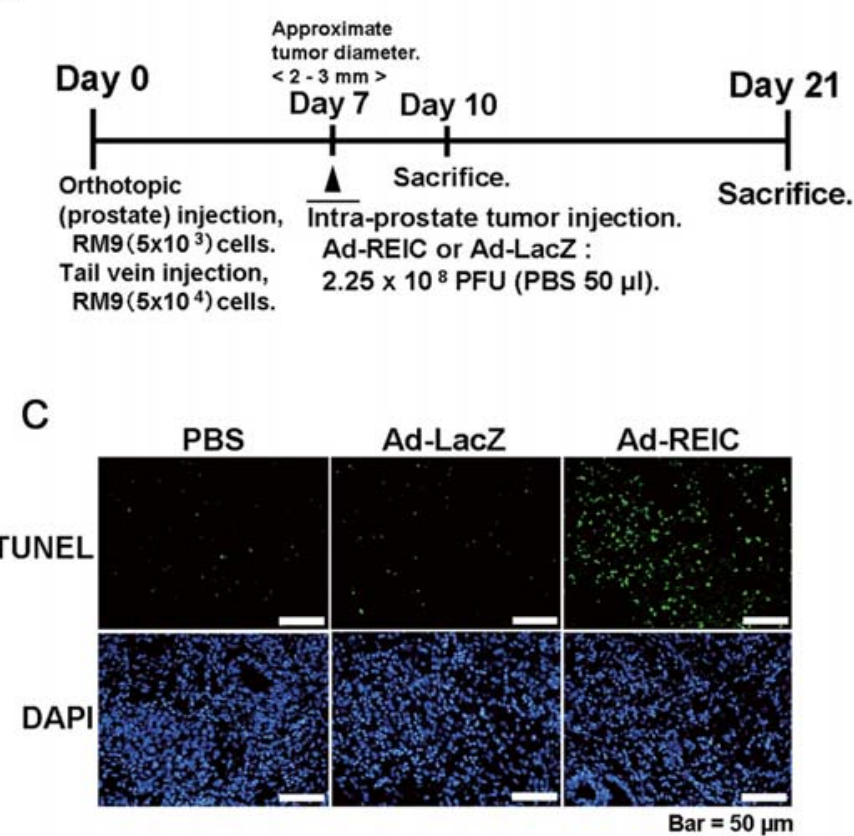

E
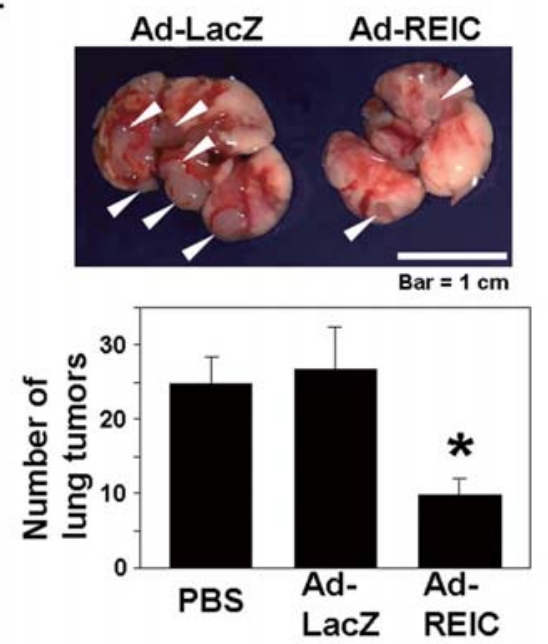

B

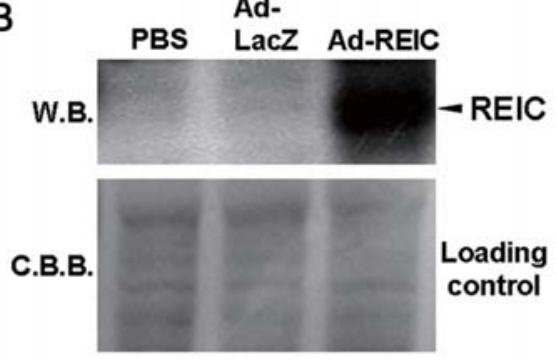

D
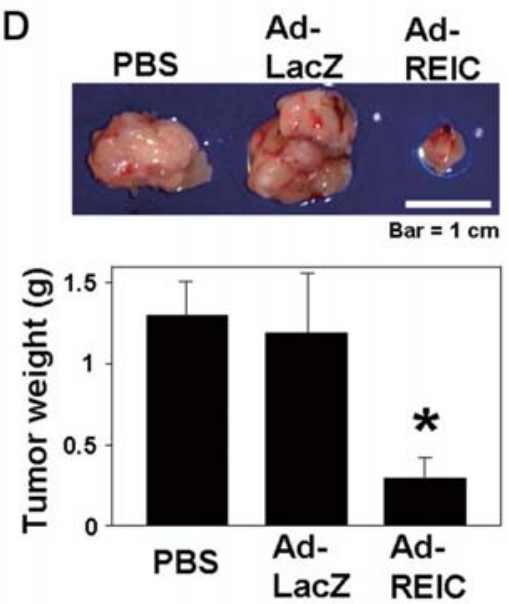

$\mathbf{F}$

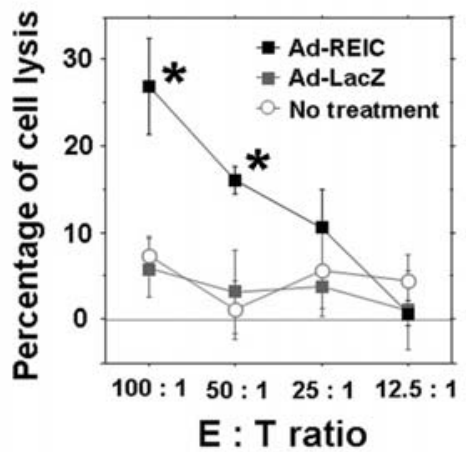

Figure 4. (A) The experimental schedule of the Ad-REIC treatment is shown. To test the intratumoral REIC/Dkk-3 expression and apoptotic induction, mice were sacrificed on day 10. In the other experiments, mice were sacrificed on day 21. (B) The expression of REIC/Dkk-3 protein in Ad-REIC injected tumors was verified by Western blotting. The gel stained with Coomassie brilliant blue is shown in the lower panel as a loading control. (C) Apoptotic induction was examined by TUNEL staining in the indicated tumors. To visualize the nuclei, 4',6-diamidino-2-phenylindol-dihydrochloride (DAPI) staining was done. (D) Macroscopic view of the orthotopic RM9 tumors on day 14 after the treatment is shown in the upper panel. The tumor weight of the RM9 tumors is shown in the lower panel. Five mice were analyzed for each group. *A significant difference between Ad-REIC and Ad-LacZ treatment. (E) Macroscopic view of metastatic RM9 lung tumors (indicated by arrowhead) on day 14 after the treatment is shown in the upper panel. The number of lung tumors is shown in the lower panel. Five mice were analyzed for each group. *A significant difference between Ad-REIC and Ad-LacZ treatment. (F) An in vitro RM9-cytolytic assay was performed using the splenocytes from each treated mouse. In the indicated effector/target (E:T) ratio, the percentage of the lysed cells was calculated. *A significant difference between Ad-REIC and Ad-LacZ treatment. Four mice were analyzed for each group.

activated STAT families tends to differ between these cell types. Further studies are therefore necessary to determine the location of REIC/Dkk-3Mo in the monocyte differentiation pathway. It is also understood that the differentiation of monocytes is intricately regulated by the interleukin and interferon cytokine family $(10,11)$. Furthermore, a specific cytokine treatment induces the activation of STAT family protein to induce the cellular differentiations $(16,17)$. Therefore, exogenous REIC/Dkk-3 protein might possess a cytokine-like function through the activation of STAT 1 and STAT 3 during the REIC/Dkk-3 Mo differentiation.

This study further demonstrated the in vivo anti-tumor effects of REIC/Dkk-3 protein to be accompanied by the up-regulation of systemic immunity against RM9 cancer cells. These findings are consistent with the significant cell death and infiltration of immunological $\mathrm{CD} 11 \mathrm{c}^{+}$and $\mathrm{CD} 8^{+}$cells observed in the treated tumor tissues. There was a prominent accumulation of CD11c positive cells, thus indicating the 
possibility that REIC/Dkk-3 protein may also differentiate mouse monocytes into the $\mathrm{CD} 11 \mathrm{c}^{+}$dendritic-like phenotype observed under in vitro conditions. It is also conceivable that the subsequent cross-talk between the CD11 $\mathrm{c}^{+}$cells and $\mathrm{CD}^{+} \mathrm{T}$ lymphocytes might stimulate anti-cancer immunity. With regard to the cytotoxic effects of the REIC/Dkk-3 protein itself, in vitro preliminary experiments were performed to examine the direct effects in RM9 cells. Even at the concentration of $20 \mu \mathrm{g} / \mathrm{ml}$, there were no significant cytotoxic changes associated with REIC/Dkk-3 treatment. Therefore, the in vivo anti-tumor effect of REIC/Dkk-3 protein largely depends on the induction of enhanced systemic anti-cancer immunity. When the orthotopic RM9 prostate tumor was treated with Ad-REIC, a significant tumor reduction was observed with local apoptotic induction. The systemic immunity against RM9 cancer cells was enhanced in the Ad-REIC treated group, thus suggesting the intratumoral overexpression of REIC/Dkk-3 protein and the local apoptotic cell death to possibly trigger the immunological modulation. Moreover, the distant anti-tumor effects in lung metastatic lesions were observed as well as at the primarily injected tumor site. Taken together, the in vitro monocyte differentiation by REIC/Dkk-3 protein into the dendritic phenotype and the in vivo results strikingly indicate that either exogenous or secreted REIC/Dkk-3 protein at the tumor site might activate the immune cells and thereby enhance the anti-cancer immunity.

The findings described herein shed new light on the functions of REIC/Dkk-3 protein in connection with both monocyte differentiation and anti-cancer immune processes.

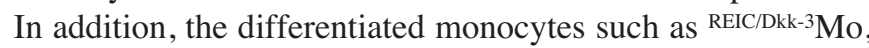
appear to be involved in the anti-cancer immunity. Therefore, REIC/Dkk-3 is an attractive immunological target which may help in the development of novel therapeutical strategies against human malignancies.

\section{Acknowledgements}

This work was supported by a grant from the Ministry of Education, Culture, Sports, Science and Technology's FY2006 'Creation of Innovation Centers for Advanced Interdisciplinary Research Areas' Scheme in Japan. We thank Katsuo Ohno and Hideo Ueki (Okayama University) for their technical assistance.

\section{References}

1. Tsuji T, Miyazaki M, Sakaguchi M, Inoue $\mathrm{Y}$ and Namba M: A REIC gene shows down-regulation in human immortalized cells and human tumor-derived cell lines. Biochem Biophys Res Commun 268: 20-24, 2000.
2. Tsuji T, Nozaki I, Miyazaki M, Sakaguchi M, Pu H, Hamazaki Y, Iijima O and Namba M: Antiproliferative activity of REIC/Dkk-3 and its significant down-regulation in non-small-cell lung carcinomas. Biochem Biophys Res Commun 289: 257-263, 2001.

3. Hsieh SY, Hsieh PS, Chiu CT and Chen WY: Dickkopf-3/REIC functions as a suppressor gene of tumor growth. Oncogene 23: 9183-9189, 2004.

4. Abarzua F, Sakaguchi M, Takaishi M, Nasu Y, Kurose K, Ebara S, Miyazaki M, Namba M, Kumon $\mathrm{H}$ and Huh NH: Adenovirus-mediated overexpression of REIC/Dkk-3 selectively induces apoptosis in human prostate cancer cells through activation of c-Jun-NH2-kinase. Cancer Res 65: 9617-9622, 2005.

5. Tanimoto R, Abarzua F, Sakaguchi M, Takaishi M, Nasu Y, Kumon $\mathrm{H}$ and Huh NH: REIC/Dkk-3 as a potential gene therapeutic agent against human testicular cancer. Int J Mol Med 19: 363-368, 2007.

6. Kashiwakura Y, Ochiai K, Watanabe M, Abarzua F, Sakaguchi M, Takaoka M, Tanimoto R, Nasu Y, Huh NH and Kumon H: Down-regulation of inhibition of differentiation-1 via activation of ATF3 and Smad regulates REIC/Dickkopf-3induced apoptosis. Cancer Res (In press).

7. Wong YC, Wang X and Ling MT: Id-1 expression and cell survival. Apoptosis 9: 279-289, 2004.

8. Glinka A, Wu W, Delius H, Monaghan AP, Blumenstock C and Niehrs C: Dickkopf-1 is a member of a new family of secreted proteins and functions in head induction. Nature 391: 357-362, 1998

9. Kawano Y, Kitaoka M, Hamada Y, Walker MM, Waxman J and Kypta RM: Regulation of prostate cell growth and morphogenesis by Dickkopf-3. Oncogene 25: 6528-6537, 2006.

10. Zhou LJ and Tedder TF: CD14 ${ }^{+}$blood monocytes can differentiate into functionally mature $\mathrm{CD} 83^{+}$dendritic cells. Proc Natl Acad Sci USA 93: 2588-2592, 1996.

11. Zou GM and Tam YK: Cytokines in the generation and maturation of dendritic cells: recent advances. Eur Cytokine Netw 13: 186-199, 2002.

12. Huang P, Watanabe M, Kaku H, Kashiwakura Y, Chen J, Saika T, Nasu Y, Fujiwara T, Urata Y and Kumon H: Direct and distant antitumor effects of a telomerase-selective oncolytic adenoviral agent, OBP-301, in a mouse prostate cancer model. Cancer Gene Ther 15: 315-322, 2008.

13. Thompson TC, Southgate J, Kitchener G and Land H: Multistage carcinogenesis induced by ras and myc oncogenes in a reconstituted organ. Cell 56: 917-930, 1989.

14. Nasu Y, Bangma CH, Hull GW, Lee HM, Hu J, Wang J McCurdy MA, Shimura S, Yang G, Timme TL and Thompson TC: Adenovirus-mediated interleukin-12 gene therapy for prostate cancer: suppression of orthotopic tumor growth and pre-established lung metastases in an orthotopic model. Gene Ther 6: 338-349, 1999.

15. Edamura K, Nasu Y, Takaishi M, Kobayashi T, Abarzua F, Sakaguchi M, Kashiwakura Y, Ebara S, Saika T, Watanabe M, Huh NH and Kumon H: Adenovirus-mediated REIC/Dkk-3 gene transfer inhibits tumor growth and metastasis in an orthotopic prostate cancer model. Cancer Gene Ther 14: 765-772, 2007.

16. Ihle JN: The Janus protein tyrosine kinase family and its role in cytokine signaling. Adv Immunol 60: 1-35, 1995.

17. Nosaka $T$ and Kitamura T: Janus kinases (JAKs) and signal transducers and activators of transcription (STATs) in hematopoietic cells. Int J Hematol 71: 309-319, 2000. 